\title{
DOI: https://doi.org/10.46296/gt.v4i8edesp.0042
}

\section{ESTRÉS LABORAL Y CLIMA ORGANIZACIONAL EN EL PERSONAL OPERATIVO DEL CUERPO DE BOMBEROS}

\section{WORK STRESS AND ORGANIZATIONAL CLIMATE IN THE OPERATIONAL PERSONNEL OF THE FIRE DEPARTMENT}

\author{
Moreira Darwin ${ }^{1}$; Rodríguez-Álava Alexandra ${ }^{2}$ \\ ${ }^{1}$ Estudiante de la Maestría Académica con Trayectoria de Investigación en Psicología, Mención \\ Psicoterapia. Instituto de Posgrado, Universidad Técnica de Manabí, UTM. Portoviejo, Ecuador. \\ Correo: andresmoreiradomo@gmail.com. ORCID ID: https://orcid.org/0000-0002-8197-7133 \\ ${ }^{2}$ Docente de la Universidad Técnica de Manabí, UTM. Portoviejo, Ecuador. Correo: \\ leonor.rodriguez@utm.edu.ec. ORCID ID: https://orcid.org/0000-0002-3034-1311
}

\begin{abstract}
Resumen
El artículo se realiza con el objetivo de analizar el estrés laboral del personal operativo del Cuerpo de Bombero de Portoviejo y cómo esto podría estar influenciando en el clima laboral dentro de la institución. Por lo que fue necesario utilizar un método cualitativo para realizar un estudio de estas variables, a través del método descriptivo y analítico se ha podido analizar cada una de las interrogantes realizadas en el cuestionario aplicado al personal. Los resultados demostraron que le $56 \%$ presenta un nivel bajo de estrés y el $3 \%$ expresó tener un alto nivel a causa del trabajo realizado dentro de la institución. Con respecto al clima laboral el personal manifestó sentirse cómodos dentro de la institución, puesto que las personas que les dirigen son líderes comprometidos con el buen desarrollo de la institución y mantienen un alto nivel de compañerismo e inclusión entre el personal, sin embargo, un grupo minoritario expresó no sentirse muy a gusto con las actividades internas realizadas frecuentemente. Por lo que se pudo concluir que a pesar de contar un alto nivel de aceptación, es necesario tener en cuenta los valores de las personas para poder trabajar en el futuro en nuevas acciones o estrategias que puedan beneficiar al personal operativo del Cuerpo de Bomberos.
\end{abstract}

Palabras clave: Personal, Estrategias, Estrés, Clima Laboral, Satisfacción.

\begin{abstract}
The article is made with the aim of analyzing the work stress of the operational staff of the Portoviejo Fire Department and how this could be influencing the work environment within the institution. Therefore, it was necessary to use a qualitative method to carry out a study of these variables, through the descriptive and analytical method it has been possible to analyze each of the questions asked in the questionnaire applied to the staff. The results showed that $56 \%$ had a low level of stress and 3\% said they had a high level because of the work done within the institution. With regard to the work environment, the staff said they felt comfortable within the institution, since the people who lead them are leaders committed to the good development of the institution and maintain a high level of companionship and inclusion among the staff, however a minority group expressed not feeling very comfortable with the internal activities carried out frequently. So it could be concluded that despite having a high level of acceptance, it is necessary to take into account the values of people to be able to work in the future on new actions or strategies that can benefit the operational staff of the Fire Department.
\end{abstract}

Keywords: Personal, Strategies, Stress, Work Climate, Satisfaction.

Información del manuscrito:

Fecha de recepción: 13 de julio de 2021.

Fecha de aceptación: 18 de octubre de 2021.

Fecha de publicación: 12 de noviembre de 2021. 


\section{Introducción}

El Cuerpo de Bomberos es una entidad encargada de dar servicio a la sociedad específicamente en la atención de emergencias, es el equipo de primera respuesta en temas de control de fuego, catástrofes, siniestros, accidentes de tránsito, entro otros. Este organismo requiere colaboradores que permitan cumplir con sus actividades en todo momento durante las 24 horas del día; es decir, están expuestos a riesgos físicos y psicosociales, que pueden constituir a la presencia de estrés dentro del marco laboral, personal y en ocasiones llevarlo al ámbito familiar y social.

El estrés laboral y un clima organizacional dentro de la profesión de bomberos, puede llegar a convertirse en amenaza, si no se trata de forma rápida y oportuna de acuerdo a los momentos que viven diariamente. Bandura considera que los individuos aprenden de su entorno social en el que se encuentran, por ello un entorno laboral apropiado favorece las condiciones de trabajo en especial aquellas que laboran atendiendo a personas.
La literatura consultada evidencia estudios desarrollados que se relacionan con esta problemática; así, Figueroa (2009, citado en Boy, 2018) realizó un estudio en México en una empresa para disminuir 0 enfrentar el estrés que manifiesta el personal operativo, constató que existe un alto porcentaje de personal que ha padecido de estrés afectando así su salud y recomienda que las personas se interesen en el tema, promoviendo e implementando un programa que beneficie a los colaboradores y permita controlar la situación de estrés establecida.

Por otra parte Miranda (2018) realizó un estudio para diagnosticar el desgaste profesional a causa del estrés en un grupo de médicos, psicólogos, enfermeras, técnicos de laboratorios, químico biólogo, educadores, trabajo social $y$ personal administrativo que forman parte de una Fundación Preventiva del SIDA en Guatemala; estableciendo que un gran porcentaje está pasando por estrés y agotamiento emocional caracterizado por ausencia o falta de energía, entusiasmo y un sentimiento de escasez de recursos; 
por lo que el autor recomienda realizar acciones preventivas $y$ terapéuticas para tratar el desgaste profesional que en muchas ocasiones influye en el clima organizacional de las personas.

Así mismo, Hidalgo (2013) realizó un estudio doctoral en la Universidad de Loja en Ecuador, sobre el estrés laboral y su influencia en el estado emocional en el personal que labora en el Ministerio de Inclusión Económica y Social. Donde se pudo constatar que el personal presenta ansiedad; estableciendo que principal factor es el estrés laboral puesto de manifiesto por la tensión, irritabilidad y fatiga. Lo cual está ligado a la sobrecarga laboral que produce un nivel de miedo y ansiedad.

Muñoz (2018) realizó una evaluación del nivel de estrés laboral de los empleados de la empresa Multicable del Ecuador. Donde luego de un análisis se calificó como bajo el nivel de estrés laboral, haciendo de esta empresa muy buena en ámbitos de estabilidad, estructura y buen clima laboral, puesto que el liderazgo mantiene buena comunicación con los empleados y entre compañeros.
En un estudio del clima laboral en una dependencia Pública en Guayaquil, Williams (2013) pudo comprobar que la motivación en los empleados está afectada, reflejado en el desinterés en las actividades en la institución donde laboran. El estudio estableció que la falta de acercamiento, claridad y confianza por parte de las empresas al exponer los objetivos institucionales crean confusión entre los empleados.

García (2018) realizó un estudio sobre el clima laboral y su relación con el nivel de satisfacción de los empleados en el GAD de Portoviejo, Ecuador; donde pudo establecer que existe una comunicación deficiente entre los distintos niveles jerárquicos; en cuanto al liderazgo, se ha ido afectando con el paso de los años y que no se ha producido una retroalimentación oportuna que mejore las acciones del personal.

En relación con el clima organizacional, Pereda et al, (2008, citado en Almeida, 2015) menciona que "es el conjunto de percepciones que los miembros de una organización tienen acerca de la misma. Las percepciones varían en función del grupo de trabajadores al que se pregunte dentro de una 
misma organización" (p. 45). Zarco et al, (2008, citado en Almeida, 2015) en su investigación establece varios climas laborales para su estudio, como son: psicológico, agregado y colectivo.

De tal forma que, los problemas de clima organizacional, se deben tratar con las debidas precauciones, por lo general señalan la necesidad de llevar a cabo un estudio que permita obtener indicadores directos de lo que se origina en la empresa $u$ organización (Pereda et al, 2018). En el libro de (Bordas, 2016) menciona que, históricamente los trabajadores y las organizaciones han padecido cambios importantes en función del devenir económico, político y cultural, propiciando el estudio del comportamiento del ser humano en estos espacios.

El libro muestra que, mediante un buen clima organizacional, las personas pueden adoptar lo mejor de sí mismo, desarrollándose a la vez de forma profesional integrándose a grupos sociales de carácter empresarial con una misma misión; de igual forma, considera que uno de los factores básicos para generar un buen clima organizacional generando satisfacción y compromiso, es el jefe inmediato, puesto que este tiene la capacidad de influir positiva 0 negativamente entre sus empleados. Por ello, el autor considera que los líderes deben ser diestros en el trato con las personas sino quieren arriesgar su propia productividad, competitividad y repercusión en el mercado.

\section{Desarrollo}

\section{Estrés laboral}

Este se define como la contestación psicológica y física que ocurre cuando las obligaciones del trabajo no concuerdan a las capacidades, recursos 0 necesidades del trabajador (Díaz, 2015). Por su parte, Gómez (2016) afirma que el estrés se encuentra en el cerebro, que es el responsable de reconocer y responder sus distintas formas; considera que las personas que presentan un grado de estrés muestran varios síntomas: presión en el pecho, hormigueo en el estómago, sudor en las palmas de las manos, dificultad para respirar, tensión muscular, falta de apetito, fatiga, entre otros. Estas manifestaciones también pueden orientarse a nivel emocional y 
mental, como inquietud, nerviosismo, ansiedad, temor, irritabilidad, enojo, golpear artículos, pánico. Incluso pueden experimentar la sensación de no controlar sus pensamientos, dificultad para tomar decisiones, dificultad para concentrarse y para actuar rápidamente, entre otros.

El estrés como una respuesta del cuerpo a condiciones externas, que perturban el equilibrio emocional de las personas. El mismo autor expresa que el estrés al inicio no se considera como una enfermedad sino como la respuesta, tanto física como mental, a las adaptaciones y ajustes del ser humano a los diversos acontecimientos vitales. Por lo cual considera que, cualquier suceso que genere una respuesta emocional, puede causar estrés, esto incluye situaciones positivas como negativas (Rodríguez, 2008. citado por Boy, 2014)

Buendía (1993, citado en McGrath, 2013) indica que el estrés es un desequilibrio avistado entre petición y contestación, y las consecuencias que esta genera la destinan al fracaso. Bernard (2000) manifiesta que los cambios externos consiguen perturbar el organismo, siendo responsabilidad del individuo mantener la estabilidad de su medio ambiente interior, aunque se modifique el exterior. Es así que el autor manifiesta que, se debe considerar el tipo de estrés que presentan los involucrados en el análisis y cómo estos pueden estar afectando en su clima laboral.

Según los varios enfoques que se han presentado el estrés se manifiesta como un desequilibrio en las personas que lo experimentan, ya sea por condiciones laborales, familiares o ambientales; lo cual podría ser la causa un desbalance en el bienestar físico y emocional, que a la vez repercutan en el ritmo laboral o profesional de los individuos. De tal forma, se puede manifestar que el estrés es una reacción del cuerpo y la mente, ante situaciones difíciles por la que atraviesa el ser humano en varios ámbitos de su vida.

\section{Tipos y causas del estrés}

El estrés es una contestación inesperada del organismo a todo requerimiento hecha sobre él (Selye 1956, citado por Amenzua 1999). Tal contestación puede producir un período de desequilibrio corporal, 
ocasionado por un estímulo al que Selye llamó estresor. También se lo denomina "Síndrome de adaptación general", como una respuesta a un estrés, la mismas que poder ser considerada como efectivo 0 perjudicial.

López (2018) considera que el estrés no es siempre perjudicial, ya que a la falta de este puede resultar la depresión. Establece dos tipos de estrés: el positivo o eustrés; y el negativo o distrés.

Una persona con estrés positivo se caracteriza por ser creativo y motivado, es lo que hace que se conserve vivo y trabajando, expresa y manifiesta sus talentos especiales, imaginación e iniciativa; es decir, es una persona que enfrenta sus problemas y los resuelve (López, 2020).

Por el contrario, el estrés negativo provoca un exceso de trabajo no asimilable fácilmente por las personas, desencadenando un desequilibrio fisiológico y psicológico que puede disminuir considerablemente en el nivel productivo (Amencua, 1999. leído en Boy 2014), aspectos que unido a características personológicas influyen significativamente en los individuos.

Lely y Dell (2010, citado en Guachamin, 2018) cita tres tipos de estrés, entre ellos está el estrés agudo, el cual surge a partir de las exigencias y presiones del pasado reciente y las presiones anticipadas del futuro cercano; este estrés se da en pequeñas dosis, puesto que si es excesivo resulta agotador. El estrés agudo episódico, quienes lo padecen asumen varias responsabilidades que no las pueden ejecutar con la rapidez deseada ni demandada; por ello se encuentran agitadas, de mal carácter, irritables, ansiosas y tensas. Estrés crónico, este tipo de estrés desgasta a las personas que lo padecen día tras día, destruyendo su cuerpo, mente y vida; surge cuando una persona no ve salida de sus problemas, por lo que abandona la búsqueda de una solución viable y opta por alternativas, como el consumo de sustancias para controlar el estrés (Guachamin, 2018).

Por su parte la Organización Mundial de la Salud (OMS), manifiesta que el estrés laboral afecta perjudicialmente la salud psicológica y física de los recursos humanos, así 
como a la eficacia de las organizaciones para las que laboran. Una mala organización de trabajo puede provocar estrés laboral. En los resultados de las investigaciones realizadas por la OMS muestran que el tipo de trabajo que produce más estrés es aquel en que las exigencias y presiones superan los conocimientos y capacidades del trabajador. De tal forma se establece que cuanto mayor sea el apoyo que el trabajador reciba de los demás, menor será la posibilidad de que sufra estrés laboral (OMS, 2020).

\section{Características del estrés relacionadas al desempeño laboral}

La mayor parte de las causas del estrés laboral, podrían estar relacionadas con la forma en que se define el trabajo y el modo en que se gestionan las entidades. Por lo que la OMS (2020) expone las siguientes características:

- Características del puesto que pueden ser tareas simples, tediosas y triviales

- Volumen y ritmo de trabajo basado en los extremos, sí como la determinación de plazos muy estrictos.
- Horarios de trabajo poco o nada flexibles

- Participación y control; falta de control a los métodos y ritmos, así como del entorno laboral (OMS, 2020).

\section{El clima organizacional}

Llaneza (2019) explica que "el clima laboral es la apreciación de los elementos que componen la cultura de una organización a un cierto periodo que es provisto por parte de los participantes" (p. 72). Esta es una condición o propiedad del ambiente apreciado o habituado por los miembros de la organización en su comportamiento (Chiavenato 2010).

Bordas et al (2016) en su investigación presenta tres niveles en el clima laboral:

1. El clima psicológico, que hace referencia a las percepciones que tienen los individuos de su entorno

2. El clima agregado, que es el conjunto de percepciones de los individuos que pertenecen a una misma unidad identificable

3. El clima colectivo, este se hace a un grupo de personas con percepciones similares, se requiere del uso de herramientas 
estadísticas para su análisis ( $p$. 83).

Por otra parte, García (2017), refiere que existen dos climas organizacionales: autoritarios que corresponden a un ambiente donde existe una estructura rígida, con agotamiento por parte del empleador, y climas participativos con un ambiente más dinámico, orientado por metas y objetivos, lo que corresponde a que es menos autocrático que los otros sistemas.

En cuanto a las dimensiones del clima laboral influyen: la cantidad de comunicación recogida por las personas, así como la organización; la diferencia entre el volumen o cantidad de comunicación indispensables y las formas para que se cumplan; cómo están las respuestas; la diferencia entre la información recolectada y recibida de acuerdo a los grados de responsabilidad; y por último la dimensiones entre los sindicatos y la empresa (Vera, 2016).

Como aspecto importante en el análisis del clima laboral, están las consecuencias que este genera en la vida de las personas $y$ organizaciones, las que pueden ser positivas como el logro, afiliación, poder, productividad, satisfacción, adaptación e innovación, entre otros; y las negativas que se establecen con un alto nivel de rotación, el ausentismo de empleados, la poca innovación, la baja productividad, entre otros, Merizalde (2011).

En concordancia a lo descrito, surge la interrogante ¿cuáles, son los indicadores para medir el clima laboral y sus consecuencias?, según, Torres (2018) para medir el clima organizacional es necesarios conocer los indicadores que miden la satisfacción laboral, estos elementos miden tanto las emociones personales y la felicidad que se siente al desarrollar las tareas en el entorno 0 puesto de trabajo, indicadores como:

- Retención del talento, este puede suponer las ventajas competitivas que tengan unas empresas sobre otras.

- El absentismo laboral, ayuda a conocer si existen problemas de funcionamiento en la empresa. Mide la ausencia de los empleados en el trabajo.

- Duración del puesto, la fuga de talento se puede dar por el tiempo en que tenga una persona 
en el mismo puesto, por ello, se debe buscar nuevas oportunidades cuando el tiempo se alarga.

- Formación y capacitación, influye directamente en el desarrollo de la actividad de la compañía suponiendo la adquisición de conocimientos teóricos y prácticos.

- Tiempo medio por contratación, es el tiempo que pasa entre que un empleado comunica su retiro de la empresa, hasta que otro candidato empieza a laborar.

- Tiempo medio en conseguir objetivo, que mide la eficacia de los empleados

- Accidentabilidad laboral, eventos que nadie desea, pero en ocasiones suceden dentro o fuera de la empresa, y deben ser monitoreados constantemente para su prevención.

Es así que se puede expresar que, el clima laborar incide positiva y negativamente a los trabajadores, considerando que, si el clima laboral es bueno, los trabajadores realizaran sus actividades de forma satisfactoria y eficiente; sin embargo, si el clima laboral es negativo, los trabajadores se sentirán cansados, fatigados y de mal humor ocasionando que su trabajo sea ineficiente y por ende los resultados sean limitados $y$ las metas institucionales se no se cumplan en su totalidad.

\section{Personal del cuerpo de bombero}

El personal operativo del cuerpo de bombero de la ciudad de Portoviejo deberá ser capaz de:

- Trabajar en espacios pocos acondicionados con poca ventilación e iluminación.

- Evidenciar destrezas en tareas prácticas

- Mantener la tranquilidad y concentrarse en la tarea y la presión

- Comunicarse de manera asertiva con los compañeros, las víctimas y ciudadanos

- Trabajar en y con el equipo

- Respetar a las personas sin discriminación

- Seguir instrucciones

- Utilizar su iniciativa para revolver problemas emergentes 
- Pasar pruebas de aptitud y médicas

- Mantener un registro de las actuaciones y salidas realizadas (CBP, 2019)

El cuerpo de bomberos es una institución particularmente desigual al resto que conforman el sector público, por su misión y estructura organizativa, la cual se conforma de una parte administrativa y otra operativa (CBP, 2019). La parte operativa por funcionarios clasificados en dos grupos: el de oficiales y el de tropa.

Los rangos que presentan van de acuerdo a su experiencia y jerarquía, diferenciando así su autoridad, como es: Bombero, Cabo, Sargento, Suboficial, Subteniente, Teniente, Capitán, Mayor, Teniente Coronel y Coronel (CBP, 2019).

\section{El personal operativo del Cuerpo} de Bomberos y el estrés

Como se ha analizado en este trabajo, el estrés se origina a partir de determinados estímulos físicos y socio ambientales, generando demanda en los individuos. En el caso del personal que presta sus servicios en una entidad de auxilio inmediato, estos aspectos pueden darse ser desde el mismo momento en que se recibe la llamada del siniestro por parte de un ciudadano, hasta la llegada al lugar donde se produce el flagelo o la intervención del mismo. Las situaciones relacionadas con el siniestro son causas estresoras muy importantes, porque producen miedo a lo desconocido, sumado a la experiencia de acontecimientos vividos; este miedo disminuye cuando se conoce ya las características del siniestro y el bombero se prepara técnica y psicológicamente para actuar (López, 2020).

Ayuso (2001) clasifica las situaciones de estrés que puede vivir el personal operativo, en:

- Desastres

naturales:

inundaciones, terremotos, incendios.

- Desastres humanos accidentales: accidentes industriales, derrumbe de edificios, accidentes ferroviarios, de aviación de tráfico con lesiones físicas graves.

- Desastres humanos voluntarios: atentados terroristas. 
El mismo autor manifiesta que el estrés en el personal del Cuerpo de Bombero es un trauma que puede afectar a cada uno de los individuos, y esto permanecerá de acuerdo a la persistencia o gravedad que ha ocasionado el estado estresante de la persona. También se debe considerar la personalidad previa al momento que inició el estrés o trastorno que se ha desarrollado, lo cual se considera síndrome postraumático (Ayuso, 2001).

\section{El clima organizacional y el Cuerpo de Bomberos}

Según los datos proporcionado por Fabara (2020) en el Ecuador el tema del clima organizacional es una problemática que se ha abordado en los últimos años, debido a la importancia que tiene en instituciones especialmente públicas como es el Cuerpo de Bomberos a nivel Nacional. En el 2018 el Ministerio del Trabajo promulgó una normativa para la medición del Clima Laboral al servicio Público, cuyo objeto fue regular los procedimientos del clima laboral y cultura organizacional en las instituciones del país; esta normativa permitió fortalecer la mejora continua en el entorno del trabajo institucional.
No obstante, el clima organizacional que incide entre el personal del Cuerpo de bombero, es una variable que no ha sido estudiada debidamente, por lo que, los autores manifiestan que se desconoce la situación actual de componentes como la interacción, visión a resultados, confianza, estimulación, comunicación, cooperación, evaluación, estructura, responsabilidad, autonomía, deber, adaptación al cambio, concordancia, sentido de pertenencia, apertura al cambio, empuje, desarrollo, ecuanimidad, reconocimiento, personalización, interacción y entorno de trabajo, entre otros (Fabara, 2020).

Chiang (2010) en su libro relaciones entre clima organizacional y satisfacción laboral menciona que el clima organizacional se basa en las personas, conducta y sentimientos, precisándolo como el conjunto de particularidades del ambiente de trabajo. Mientras que la satisfacción laboral se concibe como un estado positivo o agradable que se crean por el efecto de las experiencias laborales del trabajador y la valoración de su trabajo (Rodriguez, 2012). 


\section{Metodología}

La metodología es descriptiva y correlacional; estableciendo la relación existente entre el estrés y el clima organizacional del personal operativo en el Cuerpo de Bomberos de la ciudad de Portoviejo.

Para medir la variable del estrés laboral, se aplicó un instrumento basado en una escala de 5 niveles jerarquizados tipo Likert, donde $5=$ nunca, $4=$ muy pocas veces, $3=$ algunas veces, $2=$ casi siempre, $1=$ siempre. Para medir la variable de clima organizacional, se aplicó un instrumento basado en una escala de 7 niveles jerarquizados tipo Likert; donde $7=$ muy satisfecho, $6=$ bastante satisfecho, 5=algo satisfecho, $4=$ =indiferente, $3=$ =algo insatisfecho, 2=bastante insatisfecho 1=muy insatisfecho. Ambos fueron aplicados al personal operativo del Cuerpo de Bomberos de Portoviejo.

Una vez recogida la información se procedió a la organización y clasificación de los resultados para proceder a un análisis de los mismos. Por ello, luego de analizar de forma independiente cada resultado se realizó un cuadro de cada variable para poder establecer tanto el nivel de estrés del personal como el tipo de clima organizacional que se mantiene actualmente en la institución.

Considerando una población de 131 funcionarios dentro de la parte operativa del Cuerpo de Bombero de Portoviejo, se dividió en dos sectores, la parte rural y urbana. Por movilidad del personal se consideró pertinente enfocarse solo a la parte urbana, la cual consta de 73 funcionarios operativos.

\section{Resultados}

En base a las encuestas realizadas a los 131 funcionarios del personal operativo del Cuerpo de Bomberos de Portoviejo, se pudo establecer que el nivel de estrés que mantiene actualmente es bajo, puesto que el $56 \%$ ha manifestado que no se sienten muy estresados en estos momentos, especialmente por la época que se está pasando, donde la mayoría de las personas se encuentran en casa y el nivel de incendios ha disminuido considerablemente. Esto se da, puesto que al no dejar las casas solas, las personas están pendientes de lo que sucede dentro de sus viviendas, limitando así la posibilidad de dejar la estufa encendida o algún 
artefacto que ocasiones un acto que ponga en riesgo sus vidas o pérdida de sus bienes.

Para llegar a estos resultados, se consideró interrogantes como: el rendir informes les causa presión, o si la misión que tiene la institución origina algún tipo de estrés. A lo que se obtuvo indicadores bajos, evidenciando así, que el personal no siente estrés al realizar este tipo de trabajos. Otra de las interrogantes fue si el grupo de trabajo se encuentra organizado o si siente que el trabajo se realiza organizadamente bajo una supervisión adecuada por los superiores; a lo que se obtuvieron resultados menores a 5, equivalentes a que los investigados pocas veces sienten una presión desfavorable al realizar su labor dentro de la institución.

Al indagar sobre el clima laboral, se pudo conocer que el personal operativo presenta altos niveles de satisfacción, puesto que las personas que lo conforman y dirigen, son individuos preparados que dinamizan la labor que se realiza dentro de la institución; además el personal que labora siempre está presto en ayudarse entre ellos mismos, a fin de mantener un equipo dinámico en todo momento dentro y fuera del lugar donde se desarrollan los incendios. Esto permite que al momento de ocurrir la llamada de emergencia, todos están coordinados y actúan rápidamente para cubrir la emergencia en el menor tiempo posible, originando agilidad y oportuna actuación para salvar la vida de los ciudadanos.

Para conocer a fondo sobre el clima laboral del personal operativo se indagó sobre si los miembros del grupo son distantes, a lo que se obtuvo como respuesta que nunca sucede eso, puesto que todos colaboran y se organizan en cada actividad. Indagando un poco más, se conoció que esto se realiza a través de actividades coordinadas, con el propósito de mantener una armonía entre todos, así al momento de realizar una actividad fuera del confort de la institución, estos actúen de forma ordenada según se requiera.

\section{Discusión}

De acuerdo a la investigación teórica y práctica realizada, se considera que lo expuesto por Rodríguez 
(2008) donde menciona que cualquier suceso que genere una respuesta emocional puede ser causa de estrés; puesto que el personal operativo del Cuerpo de Bombero se encuentra siempre en constante suceso, los cuales pueden generar estrés en función de la actividad realizada diariamente. No obstante, con el bajo nivel de llamadas de emergencias en el último año fiscal, el personal ha podido relajar su condición actual emocional.

De tal forma se concuerda con lo expuesto por Bernard (2000), donde sugiere que los cambios externos pueden perturbar el organismo y tienen el poder de estabilizar al ser humano según sea su estado. Esto se comprobó mediante la aplicación del instrumento de recolección de la información, donde se conoció que a partir de que las personas se encuentran en sus hogares de forma permanente, se ha disminuido el número de llamadas de emergencias por incendios o actividades que ameriten que el personal del cuerpo de bombero acuda con urgencia al lugar, ocasionando que el personal se encuentre tranquilo y haya podido reducir individualmente el nivel de estrés que en algún momento se pudo vivir a causa de emergencias externas ocurridas en la ciudad.

En lo referente al clima laborar se pudo conocer que este es aceptable por la mayoría de las personas consideradas en la investigación, puesto que al mencionar que las autoridades son líderes que están comprometidos con el trabajo y que mantienen una relación acorde a las actividades desarrolladas diariamente, se concuerda con lo mencionado por García, donde manifiesta que los climas laborales pueden ser autoritarios y participativos, y esto influye notablemente con el ambiente interno de toda empresa $u$ organización.

\section{Conclusiones}

Luego de realizado el respectivo estudio, se puede concluir que el personal operativo del Cuerpo de Bombero de Portoviejo, mantiene un bajo nivel de estrés a causa del arduo trabajo que realiza diariamente en la ciudad, sin embargo se evidenciaron valores considerables de estrés en algunos encuestados, por lo que se considera importante que a pesar de 
no ser un número alto de bomberos afectados, se tomen las respectivas pautas para trabajar con estas personas que pueden estar propensos a una vulnerabilidad mayor y sufrir las consecuencias del estrés en un grado mayor. De forma general se puede manifestar que el personal está apto para seguir laborando en las emergencias que se susciten en la ciudad.

Tomando en cuenta los niveles de satisfacción del clima laboral, hay un mayor porcentaje de personal operativo que han mencionado sentirse satisfactoriamente en sus puestos y consideran el clima de trabajo como adecuado a sus exigencias; no obstante, hay una cantidad de bomberos que han considerado que el clima laboral no siempre es adecuado, manifestando una gran inconformidad en el área de trabajo, así también como el compañerismo, liderazgo y el trato por los superiores.

\section{Bibliografía}

Amenzua, C. (1999). Estrés, prevención y control, aprenda a relajarse. España: Ed. San Pablo. primera edición.
Ayuso, J. (2001). Trastornos de Angustia. Martínez Roca S.A.

Bernard. (2000). Cambios extremos en el ambiente perturban el organismo. Obtenido de http://evaluacioninstitucional.i doneos.com/index.php/34557 7

Bordas, M. (2016). Gestión estratégica del clima laboral.

Boy, M. E. (2014). Niveles de Estrés Laboral en los Bomberos voluntarios de la XI Compañía de Retalhuleu. Retalhuleu: Universidad Rafaél Landivar.

CBP. (2019). Modelo de Gestión del cuerpo de Bomberos Portoviejo. Obtenido de http://www.bomberosportoviej o.gob.ec/wpcontent/uploads/2019/02/MO DELO- DE-GESTION.pdf

Chiang, M. M. (2010). Clima organizacional y satisfacción laboral. Pontifica ICAI ICADE MADRID.

Chiavenato, I. (2019). Administración de Recursos Humanos. México.

Díaz, D. (2015). Estrés laboral y la morbilidad psiquiátrica entre los estudiantes de medicina que entran en la formación clínica. Bmc Medical Education. Escotolmo. Suecia., 6-14.

Fabara, G. J. (2020). Digital Publisher. Obtenido de Clima Organizacional y la satisfacción laboral en los 
empleados de la Empresa

Municipal Cuerpo de

Bomberos de Ambato:

https://www.593dp.com/index .php/593_Digital_Publisher/ar ticle/view/255/553

García, D. (2017). Estudio del clima laboral y su relación en el nivel de satisfacción de los empleados del Gad Portoviejo. Portoviejo: ULEAM.

García, D. (2018). Estudio del clima laboral y su relación con el nivel de satisfacción de los empleados del Gad Portoviejo. Universidad Eloy Alfaro de Manabí.

Gómez, P. H. (2016). Factores de Riesgo Psicosocial y Satisfacción Laboral en una Empresa. Obtenido de http://www.cienciaytrabajo.cl/ cytqa/Paginas/Factores-deRiesgo-Psicosocial-ySatisfacci\%C3\%B3n-Laboralen-una-Empresa-Chilenadel-\%C3\%81rea-de-laMiner\%C3\%ADa.aspx

Guachamin, H. (2018). Estudio de la influencia de los riesgos psicosociales en el desempeño laboral del personal de atención de emergencia del Cuerpo de Bomberos. Escuela Politécnica Nacional.

Hidalgo, D. (2013). Estrés laboral y su influencia en el Estado Emocional en el personal que labora en el Ministerio de
Inclusión Económica y social MIES. Loja: Universidad Nacional de Loja.

Llaneza, F. (2019). Ergonomía y Psicología aplicada. Manual para la formación del especialista. Lex Nova.

López. (2020). Escuela de seguridad pública del ayuntamiento de málaga.

López, J. A. (2018). Estrés y actuaciones de bomberos. Obtenido de Escuela de seguridad pública del ayuntamiento de Málaga: https://espam.malaga.eu/cata logo/BOLETINES/BOLETIN1 4/DOCS/ART0314. PDF

Marulanda, C. (2017). Estrés laboral, enemigo silencioso de la salud mental y la satisfacción con la vida. BogotáColombia.: Universidad de los Andes, Colección Prometeo.

McGrath. (2013). El estrés es desequilibrio mental. Obtenido de http://visionpsicologica.blogsp ot.com/2008/04/estresconciencia-deltiempo.html

Merizalde, S. (2011). Adaptación del a Escala del Estrés laboral para trabajadores mexicanos. Revista Salud Publica y Nutrición.

Miranda, C. (2018). Diagnóstico del nivel de desgaste del equipo multidisciplinario que trabaja en la fundación preventiva del 
SIDA. Universidad Rafael Ladivar.

Muñoz, B. (2018). Evaluar el Nivel de Estrés Laboral de los Empleados de la Empresa Multicable del Ecuador. Quito: Universidad Central del Ecuador.

OMS. (2020). La organización del trabajo y el estrés. Obtenido de Serie protección de la salud de los trabajadores: https://www.who.int/occupatio nal_health/publications/pwh3 sp.pdf?ua $=1$

Pereda, T. B. (2018). El clima organizacional y su relación con la calidad de los servicios públicos de salud: diseño de un modelo teórico. Revista Estudios Gerenciales. Obtenido de https://www.redalyc.org/pdf/2 12/21233043002.pdf

Torres, R. (2018). Indicadores clave para medir el clima laboral. Obtenido de https://fococonsultores.es/indi cadores-clave-para-medir-elclima-laboral/

Rodríguez, K. (2012). El concepto de satisfacción en el trabajo y su proyección en la enseñanza. Revista de Currículum y Formación de Profesorado, vol. 6. pp. 1-10. https://www.redalyc.org/pdf/5 67/56751267012.pdf.

Vera, J. (2016). Diseño de estrategias de comunicación para mejorar el clima laboral del cuerpo de Bomberos. Universidad de Guayaquil.

Williams, L. (2013). Diagnóstico de clima laboral en una dependencia pública. Universidad de Guayaquil. 